Ann. Biol. anim. Bioch. Biophys., I972, 12 (2), 28I-288.

\title{
PROTÉOLYSE PEPSIQUE D'ALIMENTS D'ORIGINE ANIMALE ET VÉGÉTALE
}

\author{
Marie-Claire CAMUS et Cl. SAUTIER \\ Laboratoire de Nutrition humaine, INSERM (U. 1) \\ Directeur: J. TrÉmolières \\ Hôpital Bichat, Paris, XVIII ${ }^{\mathrm{e}}$
}

\section{RÉSUMÉ}

La cinétique de protéolyse pepsique de divers aliments (blés durs, blés tendres, arachide, viande, lait) et de protéines natives et chauffées (caséine, gluten de blé) a été étudiée pendant 2 heures. La réaction enzymatique a lieu à $37^{\circ} \mathrm{C}$, à $\mathrm{pH}=\mathrm{I}, 9$, avec des quantités isoazotées de substrat. Elle est arrêtée par précipitation perchlorique à 0,5 , 10, 15, 30, 60, 120 minutes. Après filtration, on dose par la méthode de KJELDAHL l'azote acido soluble.

En début de réaction, la protéolyse est plus importante pour les aliments d'origine animale et décroît dans l'ordre suivant : viande, lait, blés tendres, arachide, blés durs. Les protéines isolées (caséine, gluten) sont hydrolysées plus rapidement et plus complètement que les aliments correspondants. La protéolyse de la caséine est supérieure à celle du gluten. Après chauffage à $100^{\circ} \mathrm{C}$ pendant I 5 minutes, seule la caséine a une protéolyse augmentée.

L'observation de la phase initiale de la réaction est importante pour différencier les substrats étudiés. La connaissance insuffisante des divers facteurs impliqués et de leurs interactions en milieu complexe, ne permet pas d'expliquer actuellement les différences observées.

\section{INTRODUCTION}

La digestibilité des aliments protéiques se mesure habituellement par la méthode des coefficients d'utilisation digestive (CUD). C'est une donnée globale qui intègre tous les constituants de la ration et toutes les étapes de la digestion. Les facteurs qui modifient le plus les CUD apparents sont les polyosides complexes des sons, (JACQUOT et GUIL,EMET r944). Avec des régimes acceptables pour 1'Homme, c'est-à-dire où l'on ne contrôle que $75 \mathrm{p}$. Ioo de la ration, et avec des aliments usuels, les CUD varient plus selon le sujet et sa période métabolique qu'avec la nature des aliments (TRÉMOLIÈRES et al., I96I). 
Or, certains aliments d'origine végétale comme les légumineuses provoquent chez 1'Homme dans l'heure qui suit leur ingestion une sensation de lourdeur digestive. Il est donc intéressant d'analyser les facteurs de digestibilité globale et tout d'abord les modalités de digestion gastrique.

GuPTA et al. (I958) observent chez le Rat une vitesse de disparition de l'azote stomacal rapide et identique pour les protéines de viande, de caséine et un mélange d'acides aminés, sauf pour la zéine où elle est très lente.

Des résultats parallèles sont obtenus par RoGERs et al. (I960) pour la zéine. Par contre, ils constatent pour la gliadine, la gélatine et un mélange d'acides aminés une évacuation stomacale analogue, plus rapide que celle des viandes cuites de bœuf et surtout de porc, et aussi que celle de la caséine qui est intermédiaire. Le temps de séjour gastrique rendrait compte de la solubilisation de 1'aliment qui ne représente qu'une phase préliminaire de la protéolyse.

In vivo, l'influence de sécrétions endogènes et la multiplicité des facteurs mis en jeu ne permettent pas d'apprécier exactement l'action des enzymes protéolytiques. On a donc été amené à comparer les protéolyses in vitro de divers substrats.

Moutonnet (I967), SUScheterT (I969), constatent après une attaque pepsique de 30 minutes une libération d'azote aminé plus importante pour le gluten que pour les protéines de lait et d'œuf. Par contre, pour Proksova et JANCARIK (I967), il n'y a plus de différence entre protéines animales et végétales, soumises à l'action de la pepsine pendant I2 heures. Mais ceci pourrait résulter d'une inhibition de la protéolyse par les produits de la réaction. Certains auteurs les éliminent par dialyse, (MAURon et Mo'TTU, I955) ou par filtration surgel en continu, (Ford rg66). Pour tenter d'expliquer ces discordances, nous avons suivi in vitro la cinétique de protéolyse pepsique de divers aliments (blés durs, blés tendres, arachide, viande, lait). Nous avons aussi étudié certaines de leurs protéines (caséine, gluten) et 1'action de la chaleur sur celles-ci.

\section{MATÉRIEL ETT MÉTHODES}

Le tableau I donne les caractéristiques des substrats étudiés.

Les blés (grains entiers) sont préalablement broyés et la viande est homogénéisée au Turax pendant 5 minutes à $4^{\circ} \mathrm{C}$. Les divers produits sont mis en solution et en suspension en milieu chlorhydrique à pH r,9, ce qui correspond à l'optimum de $\mathrm{pH}$ pour la gluténine, (OkA et al., I965), la caséine et la plupart des protéines (KRATzer et PORTER, I962).

La caséine et le gluten sont éventuellement chauffés en milieu aqueux pendant ${ }_{5}$ minutes à $100^{\circ} \mathrm{C}$.

La pepsine cristallisée Worthington, titre 2600 unités par mg. Pour un volume final de $3 \mathrm{ml}$, le milieu réactionnel renferme :

$$
\begin{array}{ll}
\text { substrat } & 2,66 \mathrm{mg} \text { d'azote par } \mathrm{ml} \\
\text { enzyme } & 0,66 \mathrm{mg} \text { par } \mathrm{ml} \text {, ou } \\
& 0,04 \mathrm{mg} \text { par } \mathrm{ml}
\end{array}
$$

La réaction a lieu en tubes à essai à $37^{\circ} \mathrm{C}$, avec agitation magnétique. Aux temps o, 5, Io, I $5.30,60$, I 20 minutes, les protéines non dégradées sont précipitées par addition de $12 \mathrm{ml} \mathrm{d}^{\prime} \mathrm{HClO}_{4}$ 2,5 p. Ioo. Après filtration, on détermine les produits d'hydrolyse en dosant l'azote du filtrat par la méthode de Kjeldahl. 


\begin{tabular}{|c|c|}
\hline Substrats & $\begin{array}{l}\text { Teneur en azote } \\
\text { p. } 100 \text { de poids frais } \\
\text { (méthode de Kjeldahl) }\end{array}$ \\
\hline Blés durs : & \\
\hline 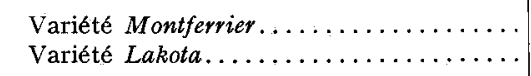 & $\begin{array}{l}1,69 \\
1,49\end{array}$ \\
\hline Blés tendres : & \\
\hline 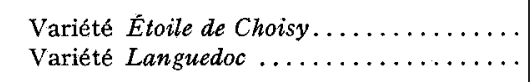 & $\begin{array}{l}1,47 \\
1,49\end{array}$ \\
\hline 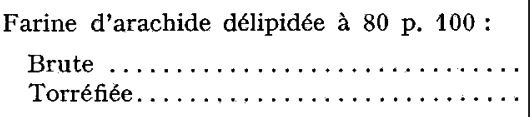 & $\begin{array}{l}7,69 \\
7,69\end{array}$ \\
\hline Muscle de bœuf (rond) $\ldots \ldots \ldots \ldots \ldots$ & 3,40 \\
\hline Lait pasteurisé $\ldots \ldots \ldots \ldots \ldots \ldots \ldots \ldots$ & 0,53 \\
\hline Caséine Hammarsten (Merck) ...... & 13,78 \\
\hline 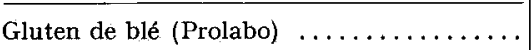 & 12,85 \\
\hline
\end{tabular}

\section{RÉSULTATS}

Les résultats (tab1. 2, 3) sont exprimés en azote soluble dans l'acide perchlorique p. roo d'azote total. Les valeurs de la protéolyse sont des valeurs moyennes suivies de leur erreur-type.

La méthode de calcul est celle des blocs casualisés (Lowy et Manchon, I969), que nous avons utilisée après vérification de l'homogénéité des variances.

Les calculs sur des rapports ont pu êtres effectués en raison de l'existence d'un dénominateur constant. Nous avons comparé les divers groupes en déterminant le degré de confiance des différences par la valeur de $F$. Elles sont dites très significatives pour un seuil de probabilité $\mathrm{P}<0,0 \mathrm{I}$, significatives pour $\mathrm{P}<0,05$, non significatives pour $\mathrm{P}>0,05$.

Le tableau 2 montre, pour de fortes doses de pepsine, d'importantes différences entre aliments d'origine animale et végétale ainsi qu'à l'intérieur de ces deux groupes.

Dans les 15 premières minutes, les vitesses de protéolyse sont différentes les unes des autres $(\mathrm{P}<\mathrm{o}, \mathrm{OI})$ et décroissent dans l'ordre: viande, lait, blés tendres, arachide brute, blés durs.

Il n'y a pas de différence significative entre blés de même espèce. Par contre, les blés tendres sont attaqués par la pepsine plus rapidement que les blés durs $(P<0,0 I)$ La protéolyse de l'arachide brute est inférieure à celle de l'arachide torréfiée $(\mathrm{P}<0, \mathrm{Or})$ et intermédiaire entre celles des blés tendres et celles des blés durs. 


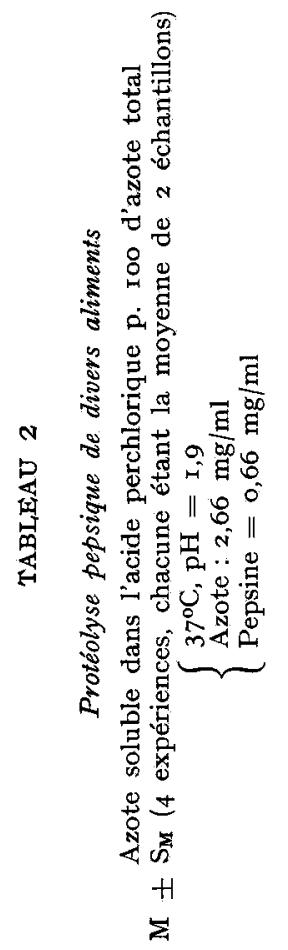

\begin{tabular}{|c|c|c|c|c|c|c|c|c|}
\hline \multirow{2}{*}{ 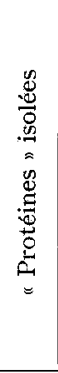 } & \multicolumn{2}{|r|}{$\frac{5}{5}$} & $\begin{array}{l}\mathbb{0} \\
0 \\
0 \\
-H \\
0 \\
0 \\
0 \\
0\end{array}$ & $\begin{array}{l}20 \\
= \\
+1 \\
\infty \\
0 \\
0\end{array}$ & 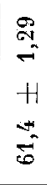 & $\begin{array}{l}\stackrel{+}{-} \\
+ \\
+1 \\
+ \\
0\end{array}$ & $\begin{array}{l}\overrightarrow{0} \\
\stackrel{5}{0} \\
H \\
H \\
\infty \\
0 \\
0\end{array}$ & $\begin{array}{l}\overrightarrow{0} \\
\infty \\
0 \\
0 \\
H \\
H \\
\infty \\
\infty \\
\stackrel{0}{1}\end{array}$ \\
\hline & & $\begin{array}{l}\text { ON } \\
0 \\
0 \\
\text { ti } \\
0 \\
0 \\
0 \\
0 \\
0\end{array}$ & $\begin{array}{l}\stackrel{2 g}{-} \\
+ \\
+1 \\
g \\
8\end{array}$ & 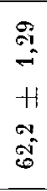 & 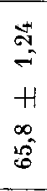 & $\begin{array}{l}\text { : } \\
5 \\
8 \\
H \\
H \\
8 \\
8 \\
8\end{array}$ & $\begin{array}{l}\overrightarrow{0} \\
\infty \\
\infty \\
0 \\
H \\
\infty \\
\infty \\
\stackrel{\infty}{\infty}\end{array}$ \\
\hline \multirow{2}{*}{ 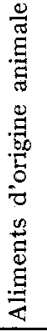 } & \multicolumn{2}{|c|}{ 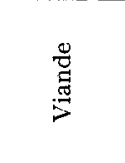 } & 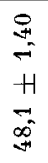 & 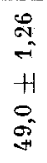 & $\begin{array}{l}8 \\
- \\
+1 \\
0 \\
0 \\
\text { in }\end{array}$ & 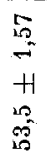 & $\begin{array}{l}8 \\
-1 \\
+1 \\
-5 \\
-5\end{array}$ & 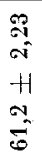 \\
\hline & \multicolumn{2}{|c|}{ 葷 } & $\begin{array}{l}\stackrel{2}{-} \\
= \\
H \\
0 \\
\infty \\
\infty \\
\infty\end{array}$ & 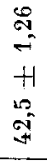 & $\begin{array}{l}8 \\
-7 \\
H \\
-1 \\
2 \\
3\end{array}$ & $\begin{array}{l}5 \\
\stackrel{6}{-6} \\
+1 \\
\infty \\
\sigma \\
\sigma\end{array}$ & $\begin{array}{l}8 \\
- \\
H \\
\text { i } \\
\text { i }\end{array}$ & $\begin{array}{l}\text { s } \\
\text { o } \\
+1 \\
\infty \\
0 \\
\text { in }\end{array}$ \\
\hline \multirow{6}{*}{ 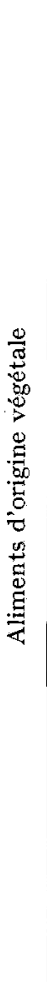 } & \multicolumn{2}{|c|}{ 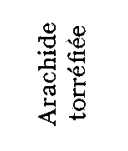 } & 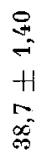 & $\begin{array}{l}\stackrel{0}{\pi} \\
\stackrel{-}{+} \\
H \\
\infty \\
\stackrel{-}{+}\end{array}$ & $\begin{array}{l}0 \\
\text { in } \\
+1 \\
\text { on } \\
\text { on } \\
\text { on } \\
\text { a }\end{array}$ & 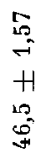 & $\begin{array}{l}8 \\
\stackrel{8}{-} \\
H \\
8 \\
0 \\
0 \\
+\end{array}$ & $\begin{array}{l}\text { of } \\
\text { of } \\
\text { H } \\
\text { of } \\
\text { of } \\
\text { is }\end{array}$ \\
\hline & \multicolumn{2}{|c|}{ 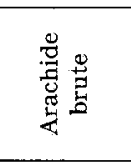 } & $\begin{array}{l}9 \\
\stackrel{+}{-} \\
H \\
0 \\
\stackrel{-}{0}\end{array}$ & \begin{tabular}{l}
$\stackrel{9}{9}$ \\
- \\
$H$ \\
0 \\
\multirow{\sigma}{*}{}
\end{tabular} & $\begin{array}{l}0 \\
- \\
H \\
0 \\
\text { S- } \\
\text { S }\end{array}$ & 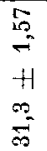 & $\begin{array}{l}8 \\
\stackrel{8}{-} \\
H \\
0 \\
0 \\
0\end{array}$ & 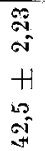 \\
\hline & \multirow{2}{*}{ 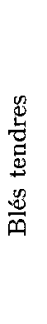 } & 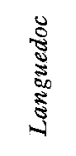 & 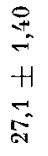 & 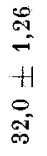 & $\begin{array}{l}0 \\
-1 \\
+1 \\
\infty \\
\infty \\
\infty\end{array}$ & 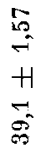 & $\begin{array}{l}8 \\
-1 \\
+1 \\
\infty \\
0 \\
0 \\
0\end{array}$ & $\begin{array}{l}9 \\
0 \\
0 \\
+1 \\
0 \\
0 \\
0\end{array}$ \\
\hline & & 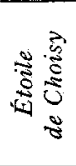 & $\begin{array}{l}\stackrel{9}{+} \\
\text { - } \\
+1 \\
\infty \\
\infty \\
\infty\end{array}$ & $\begin{array}{l}\stackrel{0}{2} \\
\stackrel{-}{+} \\
+1 \\
\vdots \\
\dot{0}\end{array}$ & 落 & $\begin{array}{l}5 \\
\text { s. } \\
-1 \\
+1 \\
0 \\
0 \\
0 \\
0\end{array}$ & 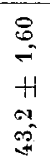 & $\begin{array}{l}\text { â } \\
\text { oi } \\
+1 \\
\text { in } \\
\text { in } \\
\text { in }\end{array}$ \\
\hline & \multirow{2}{*}{ 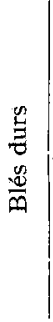 } & 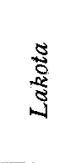 & $\begin{array}{l}\stackrel{+}{+} \\
\rightarrow-1 \\
H \\
\infty \\
\infty \\
\infty\end{array}$ & 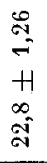 & 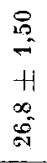 & 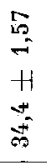 & 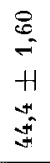 & $\begin{array}{l}\text { No } \\
\text { oi } \\
H \\
\text { H } \\
0 \\
0\end{array}$ \\
\hline & & 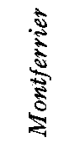 & $\begin{array}{l}\stackrel{P}{+} \\
\rightarrow \\
+1 \\
\infty \\
\infty \\
\infty\end{array}$ & 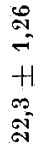 & 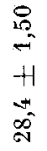 & $\begin{array}{l}\text { is } \\
\text { s. } \\
\text { H } \\
\infty \\
\text { ô }\end{array}$ & $\begin{array}{l}8 \\
- \\
+1 \\
0 \\
0 \\
0\end{array}$ & $\begin{array}{l}\text { ज̂ } \\
\text { ô } \\
\text { H } \\
\text { on } \\
\text { î }\end{array}$ \\
\hline \multicolumn{3}{|c|}{ 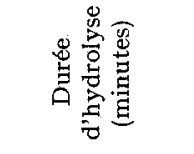 } & 10 & $\vartheta$ & $\stackrel{12}{\rightarrow}$ & శి & 8 & స్తి \\
\hline
\end{tabular}


A 60 minutes et surtout à 120 minutes, les différences entre les divers aliments s'estompent, sauf pour la viande dont la protéolyse reste supérieure et pour l'arachide brute, dont la protéolyse reste inférieure.

Les protéines isolées (caséine, gluten) sont plus rapidement hydrolysées que celles incluses dans les aliments. Pour de fortes concentrations d'enzyme $(0,66 \mathrm{mg} / \mathrm{ml})$, il n'y a de différences significatives $(P<0,05)$ entre les deux substrats qu'en début de réaction. Pour de faibles concentrations d'enzyme (0,04 $\mathrm{mg} / \mathrm{ml})$ (tabl. 3), la différence est augmentée de $20 \mathrm{p}$. Ioo en faveur de la caséine et celle-ci se maintient pendant toute la réaction.

\section{TABLEAU 3}

Protéolyse pepsique de protéines isolées

Azote soluble dans l'acide perchlorique p. roo d'azote total $\mathrm{M} \pm \mathrm{S}_{\mathrm{M}}$ (4 expériences, chacune étant la moyenne de 2 échantillons)

$$
\left\{\begin{array}{l}
37^{\circ \mathrm{C}}, \mathrm{pH}=\mathrm{I}, 9 \\
\text { Azote : } 2,66 \mathrm{mg} / \mathrm{ml} \\
\text { Pepsine : } 0,04 \mathrm{mg} / \mathrm{ml}
\end{array}\right.
$$

\begin{tabular}{c|c|c|c|c}
\hline $\begin{array}{c}\text { Durée d'hydrolyse } \\
\text { (minutes) }\end{array}$ & Caséine & $\begin{array}{c}\text { Caséine chauffée } \\
15 \text { minutes à } 100^{\circ} \mathrm{C}\end{array}$ & Gluten & $\begin{array}{c}\text { Gluten chauffé } \\
15 \text { minutes à } 100^{\circ} \mathrm{C}\end{array}$ \\
\cline { 2 - 5 } & $16,07 \pm 0,676$ & $19,40 \pm 0,676$ & $11,91 \pm 0,676$ & $12,14 \pm 0,676$ \\
\hline 5 & $21,9 \pm 1,33$ & $25,8 \pm 1,33$ & $17,8 \pm 1,33$ & $16,8 \pm 1,33$ \\
\hline 10 & $28,67 \pm 0,792$ & $31,45 \pm 0,792$ & $22,73 \pm 0,792$ & $22,17 \pm 0,792$ \\
\hline 15 & $39,2 \pm 1,91$ & $40,6 \pm 1,91$ & $27,7 \pm 1,91$ & $27,0 \pm 1,91$ \\
\hline 30 & $48,8 \pm 1,71$ & $51,0 \pm 1,71$ & $39,0 \pm 1,71$ & $34,5 \pm 1,71$ \\
\hline 60 & $57,5 \pm 1,99$ & $52,1 \pm 1,99$ & $46,0 \pm 1,99$ & $42,1 \pm 1,99$ \\
\hline 120 & & & \\
\hline \hline
\end{tabular}

Une ébullition de I5 minutes ne modifie que la phase initiale de la protéolyse de la caséine $(\mathrm{P}<0, \mathrm{OI})$.

\section{DISCUSSION}

En début de réaction, les substrats d'origine animale ont une protéolyse plus rapide que ceux d'origine végétale.

Avec les protéines incluses dans les aliments, on observe, pendant les I5 premières minutes, des différences significatives qui tendent à disparaître pour la plupart d'entre elles en fin de réaction. Avec les protéines isolées, ces différences n'existent plus après ro minutes à de fortes concentrations d'enzyme, alors qu'à de faibles concentrations de celle-ci, elles sont plus grandes et subsistent ensuite. Ces résultats sont en contradiction avec ceux de SuscheTET (Ig69) qui trouve à 30 minutes une 
hydrolyse pepsique du gluten plus élevée que celle de la caséine. Ceci peut être lié à des conditions expérimentales et à des échantillons de gluten différents. En effet, il opère avec un rapport enzyme-substrat, voisin du nôtre, mais il arrête la réaction par ébullition et neutralisation et non par un réactif précipitant des protéines. De plus, selon l'échantillon de gluten utilisé, il observe toujours à 30 minutes des protéolyses nettement distinctes qui diminuent avec le temps de stockage et se rapprochent de celle de la caséine. Or, les différences que nous constatons entre les divers substrats se manifestent surtout pendant les Io à I 5 premières minutes de la réaction, alors que le substrat est encore en excès par rapport à l'enzyme et que la pseudolinéarité des courbes permet la détermination de la vitesse initiale. Encore faut-il préciser si les différentes vitesses de protéolyse sont liées à la nature des diverses protéines ou résultent de l'interaction de nombreux facteurs présents.

Les substances associées agissent certainement. Nous observons ainsi une protéolyse plus rapide, et plus complète des protéines isolées que des aliments correspondants, soit une augmentation de $20 \mathrm{p}$. Ioo pour le gluten de blé et de I $5 \mathrm{p}$. Ioo pour la caséine de lait. Dans le cas de mouture intégrale de blé, ceci peut être dû à la forte teneur en indigestible glucidique (cellulose, hémicellulose) dont on connaît l'action dépressive sur les CUD. En plus de leur rôle sur le transit intestinal, ces substances auraient un pouvoir adsorbant (TRÉMOLIÈRES et ERFMAN, I944), et diminueraient la quantité d'enzyme disponible. Ces faits sont à rapprocher de l'action inhibitrice sur la pepsine de certains polysaccharides, capables de former un complexe avec l'enzyme (ANDRÉ, I970).

Dans le cas du lait, l'état physico-chimique de la caséine (micelles de phosphocaséinate de calcium) et ses variations de solubilité peuvent intervenir. En effet, la solubilité des protéines facilite probablement leur dégradation enzymatique en augmentant la surface d'attaque. Par chauffage de la caséine, nous observons une augmentation de la solubilité et parallèlement un accroissement de la vitesse initiale de protéolyse. Par contre, il n'y a aucun changement pour le gluten qui reste peu soluble. La chaleur entraîne en général une dénaturation des protéines pouvant modifier leur attaque enzymatique ultérieure. Cependant, dans le cas du gluten, la richesse en ponts $S-S$ particulièrement thermorésistants, pourrait limiter cette dénaturation.

Malgré une teneur identique entre les divers acides aminés (Busson et al., rg66), les blés tendres sont protéolysés plus vite que les blés durs. Ceci pourrait être dû à la dimension plus grande des particules des moutures de blé dur. Mais, des résultats inverses étant obtenus pour la protéolyse trypsique, il semble plus probable que les différences proviennent de la séquence de leurs acides aminés. Par ailleurs, des vitesses de migration électrophorétique différentes ont été mises en évidence pour certaines de leurs protéines : albumines et globulines (FEUILLET et BoURDET, I967), gliadines, (HUEBNER et RoTHFUS, I968). I1 reste difficile d'expliquer avec certitude les différences de protéolyse pepsique des substrats étudiés, notre connaissance de leur structure et des divers facteurs associés étant encore très insuffisante. Cependant, la protéolyse des protéines d'origine animale plus rapide en début de réaction, pourrait être rapprochée de la préférence que l'Homme manifeste à leur égard, sans doute due, en partie, à leur digestion gastrique plus "légère ». En admettant que roo $\mathrm{ml}$ d'un suc gastrique normal de concentration en pepsine voisine de 0,05 à $\mathrm{I} \mathrm{mg} / \mathrm{ml}$ soient en contact avec Ioo grammes de viande ou $250 \mathrm{ml}$ de lait, le rapport pepsine/azote peut varier 
de 0,004 à 0,08 . Or, dans nos expériences, ce rapport varie de 0,015 à 0,250 et peut donc être considéré comme physiologique.

Il semble donc qu'il y ait corrélation entre la plus ou moins grande vitesse initiale de protéolyse telle que nous l'avons mesurée, et la plus ou moins grande appétence de 1'Homme pour les protéines correspondantes. Par conséquent, si des études ultérieures tant in vitro qu'in vivo confirment ces résultats, il pourrait être envisagé d'inclure ce test de mesure des vitesses initiales de protéolyse pepsique parmi ceux déjà classiques concernant la digestibilité.

Reçu pour publication en janvier 1972.

\section{SUMMARY}

\section{PEPTIC PROTEOLYSIS OF ANIMAL AND VEGETABLE FOODS}

The time course of the peptic proteolysis of various foods (Triticum durum, Triticum vulgare groundnut, meat, milk) and of raw and heated proteins (casein, wheat, gluten) was followed during 2 hours.

The conditions of the enzymatic reaction were : $37^{\circ} \mathrm{C}, \mathrm{pH}, \mathrm{I}, 9$ and such quantities of substrate as to obtain a constant nitrogen concentration in all experiments. The reaction was stopped by perchloric precipitation at $0,5,10, \mathrm{I} 5,30,60$, I20 $\mathrm{mn}$. After filtration, the determination of acido-soluble nitrogen was done by the Kjeldahl method. In the early phase, proteolysis was higher for the animal foods, in the following decreasing order : meat, milk, Triticum vulgare, groundnut, Triticum durum.

Isolated proteins were hydrolyzed faster and more completely than those of the corresponding foods. Casein proteolysis was greater than gluten proteolysis. After heating at $100^{\circ} \mathrm{C}$ for I $5 \mathrm{mn}$, proteolysis was only increased for casein.

It is important to determine the first steps of the reaction to differenciate the studied substrates. The insufficient knowledge of the various involved factors and of their interactions in complex media does not allow for the time being the explanation of the observed differences.

\section{RÉFÉRENCES BIBLIOGRAPHIQUES}

André C., I970. Méthode de mesure de l'inhibition de la protéolyse pepsique par les polysaccharides et les glycoprotéines acides. C.R. Soc. Biol. Lyon, 164, 853-856.

Busson F., Fauconneau G., Pion N., Montreuil J., r966. Protides, 2 e section : acides aminés. Ann. Nutr. Alim. 20 (2), 199-2I9.

Feuillet P., Bourdet A., r967. Composition protéique et caractéristiques génétiques des blés. Bull. Soc. Chim. Biol., 49 (го), 1273-1283.

Ford J. E., Salter D. W., I966. Analysis of enzymically digested food proteins by sephadex gel filtration. Br. J. of Nutr., 20, 843-86o.

Gupta J. D., Dakroury A. M., Harper A. E., 1958. Observations on protein digestion in vivo. Rate of disappearance of ingested protein from the gastro-intestinal tract. J. of Nutr., 64, 447-456.

Huebnek F. R., Rothfus J. A., I 668 . Gliadin proteins from different varieties of wheats. Cereal chem, 45 (3), 242-253.

Jacouot R., Guillemet R., I944. Vues sur le problème du pain. Trav. Inst. nat. Hygiène, 1 (3), I $77-209$.

Kratzer F. H., Porter J. W. C., I962. The effect of $\mathrm{pH}$ on the digestion of proteins in vitro by pepsin. Brit. J. Nutr., 16, 579-584.

Lowy R., Manchon P., I969. Eléments de statistiques appliqués à la Biologie. Vol. 2, Olivetti éd., Paris.

MaURon J., Mottu F., I955. The availability of lysine, methionine and tryptophan in condensed milk and milk powder in vitro digestion studies. Arch. Biochem. Biophys., 59, 433-45I. 
Moutonnet P., I967. Influence des sulfites sur la protéolyse : étude in vitro. Ann. Biol. anim., Bioch. Biophys., 7 (1), 63-7I.

OKa S., Babel F. J., DraudT H. N., 1965. Proteolytic action of pepsin on glutenin. J. Food. Sci., 30 (2), 2 I2-2I7.

Proksova M., JANCARIK A., I967. The variability of the tryptic digestibility of vegetable proteins. Zivoc VYR I2 (XL) 479-488.

Rogers Q. R., Chen M. C., Peraino C., Harper A. E., 196o. Observations on protein digestion in vivo. Recovery of nitrogen from the stomach and small intestine at intervals after feeding diets containing different proteins. J. of Nutr., 72, 33I-339.

Suschetet M., I 969 . Influence des produits solubles formés par chauffage du glucose et du glycocolle sur l'hydrolyse enzymatique de différentes protéines. Ann. Biol. anim. Bioch. Biophys., 9 (3), 405-412.

Trémoltêres J., ERFMan R., I944. Étude sur la digestibilité du pain noir bluté à 98 p. roo. Trav. Inst. nat. Hygiène, 1 (2), 366-390.

Tremolieres J., Sautier Cl., Faudemay F., Farquet J., r96r. Effets de diverses graisses sur la digestibilité chez l'homme. Enzymes of Lipids Metabolism. Pergamon Press, 195-202. 